\title{
BIOESTRATIGRAFIA DO CRETÁCEO MARINHO DA BACIA DE CAMPOS, BRASIL
}

\author{
RICARDO LATGÉ MILWARD DE AZEVEDO* , JOSÉ GOMIDE* , \\ MARTA CLÁUDIA VIVIERS* e ARMANDO TERUO HASHIMOTO*
}

\begin{abstract}
An accurate biostratigraphic framework of the Cretaceous marine section of Campos Basin was established based on studies of foraminifera, nannotossils, and palynomorphs. With the research of 77 wells it was possible to subdivide the Albian-Masstrichtian section in 11 zones of foraminifera, 6 zones of nannofossils, and 7 zones of palynomorphs (the study of which was restricted to the Albian-Santonian stages). Some of these zones remain still informal. Three main regional unconformities were found in the biostratigraphic sequence. These gaps correspond to the Early-Middle Cenomanian, Middle-Late Turonian and Campanian. Less important hiatuses were also found in the area.
\end{abstract}

INTRODUÇÃo A seção marinha do Cretáceo "Médio." -Superior na área oriental da Bacia de Campos ainda apresenta problemas de correlação e determinação de ambientes deposicionais.

O detalhamento dessas séries, afetadas por intensa estruturação halocinética e com diversos corpos arenosos turbidíticos encaixados em seus estratos, exige a conjugação de estudos biolito e paraestratigráficos. Tal conjugação tem sido realizada pela integração dinâmica dos dados obtidos no Setor de Bioestratigrafia e Paleoecologia do Centro de Pesquisas da Petrobrás (Cenpes) com aqueles originados no Grupo de Análise da Bacia de Campos desta mesma empresa.

Este trabalho apresenta o primeiro biozoneamento integrado de nanofósseis, foraminiferos e palinomorfos específico para a Bacia de Campos.

\section{LOCALIZAÇÃo E MÉTODOS A área estudada situa-se} na parte oriental da Bacia de Campos, entre as coordenadas geográficas $40^{\circ}$ e $41^{\circ}$, de longitude oeste, e $21^{\circ}$ e $23^{\circ}$, de latitude sul. Neste polígono foram selecionados 77 poços (Fig. 1).

Nas análises bioestratigráficas foram utilizados os foraniníferos, nanofósseis e palinomorfos recuperados da coluna de sedimentos. Mais de 2.000 amostras de calha e 500 de testemunhos foram examinados, sendo boa parte delas do tipo punctual e representativa de horizontes argilosos.

ARCABOUÇO GEOLÓGICO E ESTRATIGRAFIA A Bacia de Campos, situada na margem leste da Plataforma Continental Brasileira, tem como limite setentrional o Arco de Vitória e, meridional, o Arco de Cabo Frio. Sua seção é dividida em quatro seqüências tectono-sedimentares: uma não-marinha (fase rifte); uma evaporítica; uma marinha pré-oceânica, dominantemente carbonática; e uma marinha oceânica, com predomínio de terrígenos.

As unidades litoestratigráficas que a constituem foram formalizadas por Schaller (1973). A seção analisada abrange a Formação Macaé e a porção cretácea da Formação Campos.

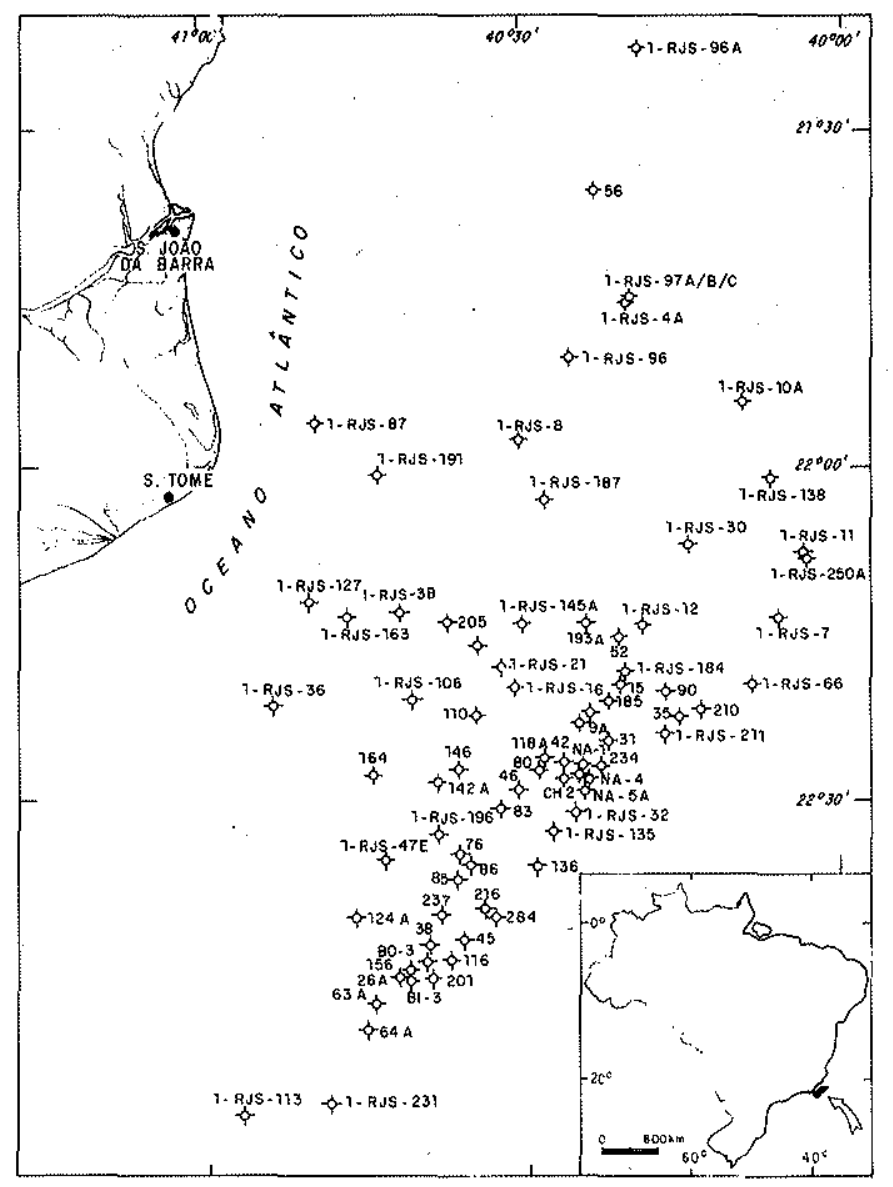

Figura 1 - Mapa de localizaçäo

O padrão estrutural e a distribuição dos reservatórios de ambas as formações foram controladas por processos de tectônica de sal Figueiredo \& Mohriak (1984). Os mesmos

\footnotetext{
** Centro de Pesquisas da Petrobrás (Cenpes), Setor de Bioestratigrafia e Paleoecologia. Cidade Universitária, Quadra 7, Prédio 20, llha do Fundāo, CEP 21910, Rio de Janeiro, RJ, Brasil
} 
autores são de opinião que a halocinese, em sua fase inicial, formou almofadas de sal que controlaram as fácies porosas dos carbonatos Macaé de águas rasas. Cóm a continuidade do processo, formaram-se falhas de crescimento, crista de roll-over e dobras anticlinais, de idades que variam do Mesocretáceo ao Terciário. Arenitos turbidíticos são freqüentes e localizam-se, em geral, em baixos formados durante a evolução das falhas de crescimento. Esses reservatórios contêm, em muitos casos, importantes acumulações de hidrocarbonetos (exemplo, Campo de Namorado).

A figura 2 apresenta o quadro esquemático cronobiolitoestratigráfico da bacia proposto neste trabalho. As zonas são apresentadas no código alfa-numérico utilizado na $\mathrm{Pe}$ trobrás.

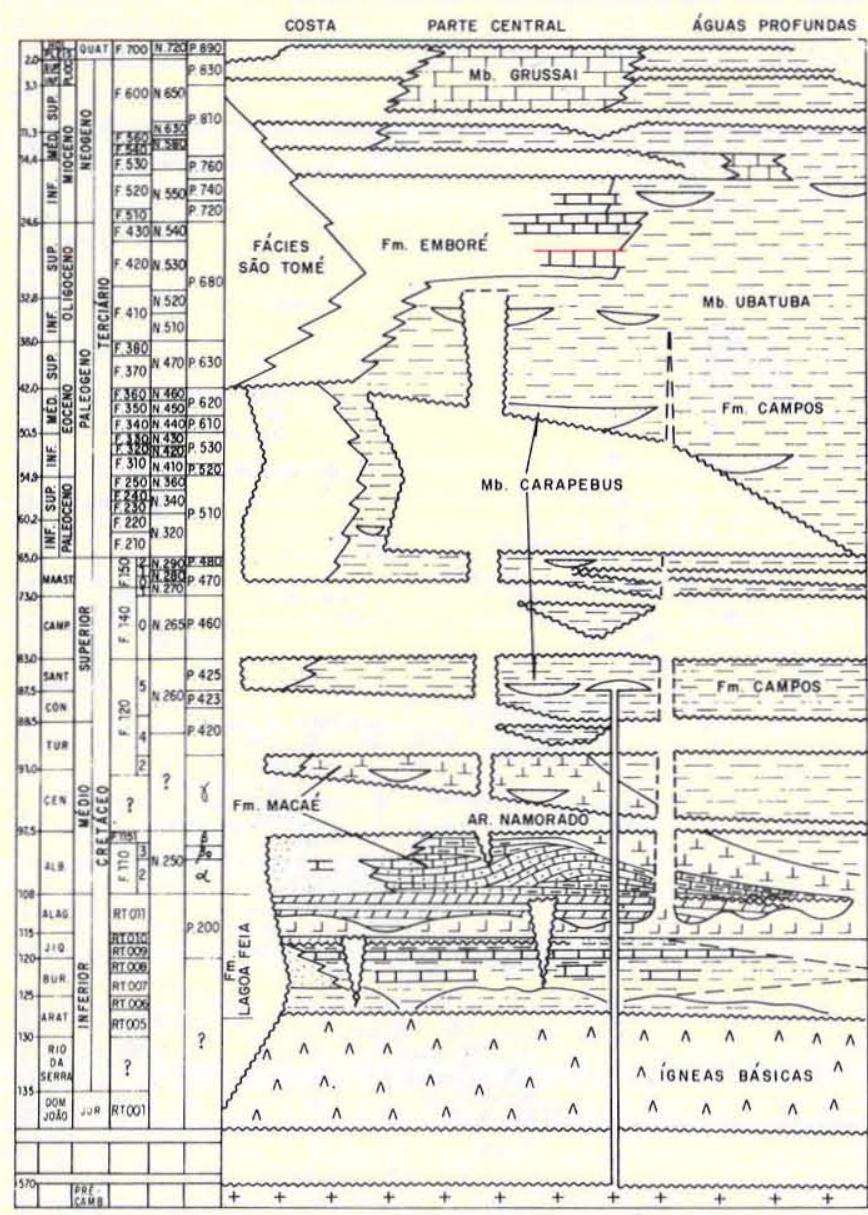

Figura 2 - Seção esquemática cronobiolitoestratigráfica modificada da coluna do Secasu-82 (escala de tempo absoluta de Harland et al. 1982, Beurlen 1981, para os andares locais)

PROPOSIÇÃO BIOCRONOESTRATIGRÁFICA A ênfase exploratória dada à Bacia de Campos após a descoberta de óleo, em 1975, em sua porção submersa, exigiu que o acompanhamento bioestratigráfico dos poços perfurados se fizesse de maneira mais rápida e eficiente. Poucos anos antes, Quadros \& Gomide (1972) haviam demonstrado o potencial dos nanofósseis como bons indicadores estratigráficos para as bacias marginais brasileiras. Além de abundantes nas rochas sedimentares, eram facilmente recuperados e descritos.

Excetuando-se a Série Cretáceo "Médio", onde se notava uma escassez de bio-horizontes nanofossilíferos, os cocolitoforídeos logo demonstraram ser a mais eficiente ferramenta de correlação e datação do pacote sedimentar marinho da bacia.

Dada a importância econômica da seção Albiano-Turoniano, a Petrobrás decidiu investir na pesquisa de outros microrganismos que permitissem subdividir esses andares. Surgiu, então, o detalhamento palinológico proposto por Uesugui (1976) e, bem mais tarde, o estudo dos calcisferulídeos da Formação Macaế (Dias-Brito 1985), que abrangem quase a totalidade desses andares.

O zoneamento apresentado neste trabalho inclui, pela primeira vez na Bacia de Campos, informações quanto à sucessão dos foraminíferos. Foram reconhecidas 11 unidades bioestratigráficas desses microfósseis e 6 de nanofósseis para o intervalo Albiano-Maastrichtiano. Os palinomorfos contribuíram no detalhamento da seção com 7 biozonas compreendidas entre o Albiano e o Santoniano. Algumas dessas bio-unidades foram descritas em zoneamentos que abrangem diversas bacias sedimentares brasileiras (Troelsen \& Quadros 1971, Noguti \& Santos 1972, Regali et al. 1974).

A seguir, são descritas as biozonas reconhecidas na bacia. Um quadro que correlaciona os três zoneamentos às unidades cronoestratigráficas internacionais é apresentado na figura 3. Quase todas as unidades representadas são do tipo zona-intervalo. Algumas delas assumem ainda um caráter informal, aguardando-se maiores estudos para sua formalização. Trata-se, de um modo geral, de zonas indicadas por taxon não muito freqüente na bacia, mas de reconhecida expressão internacional ou comum em áreas vizinhas. Há casos, também, de espécies descritas informalmente, não sendo prudente, portanto, sua utilização formalizando zonas.

Foraminíferos Os foraminíferos encontrados na Bacia de Campos estão mostrados na figura 4.

ZONA Trocholina sp. (INFORMAL) Caracterizada pela amplitude de ocorrência local de trocholina sp., esta unidade bioestratigráfica tem como formas associadas Favusella spp, e alguns ostracodes marinhos, principalmente o gênero Conchoecia. Seu limite inferior é estabelecido no horizonte afossilífero que acompanha o topo da Formação Lagoa Feia (evaporitos).

A amplitude cronoestratigráfica desta biozona não pode ainda ser definida com precisão. Ela foi associada ao Eo-Mesoalbiano, devido à concorrência das formas descritas com os palinomorfos indicativos da zona Cyclopsiella sp. Sua distribuição na bacia é limitada, concentrando-se na região norte. de.

O poço 1-RJS-76 apresenta a melhor seção desta unida-

ZONA Favusella washitensis Seu limite superior foi estabelecido ao nível de extinção local da espécie Favusella washitensis. Sua base foi fixada no nível de desaparecimento das formas diagnósticas da zona Trocholina sp. Modifica-se, neste ponto, a definição proposta por Viviers (1982) para a bio-unidade homônima que ocorre no Albiano da Bacia do Ceará.

Ela corresponde à Zona Hedbergella washitensis de No- 


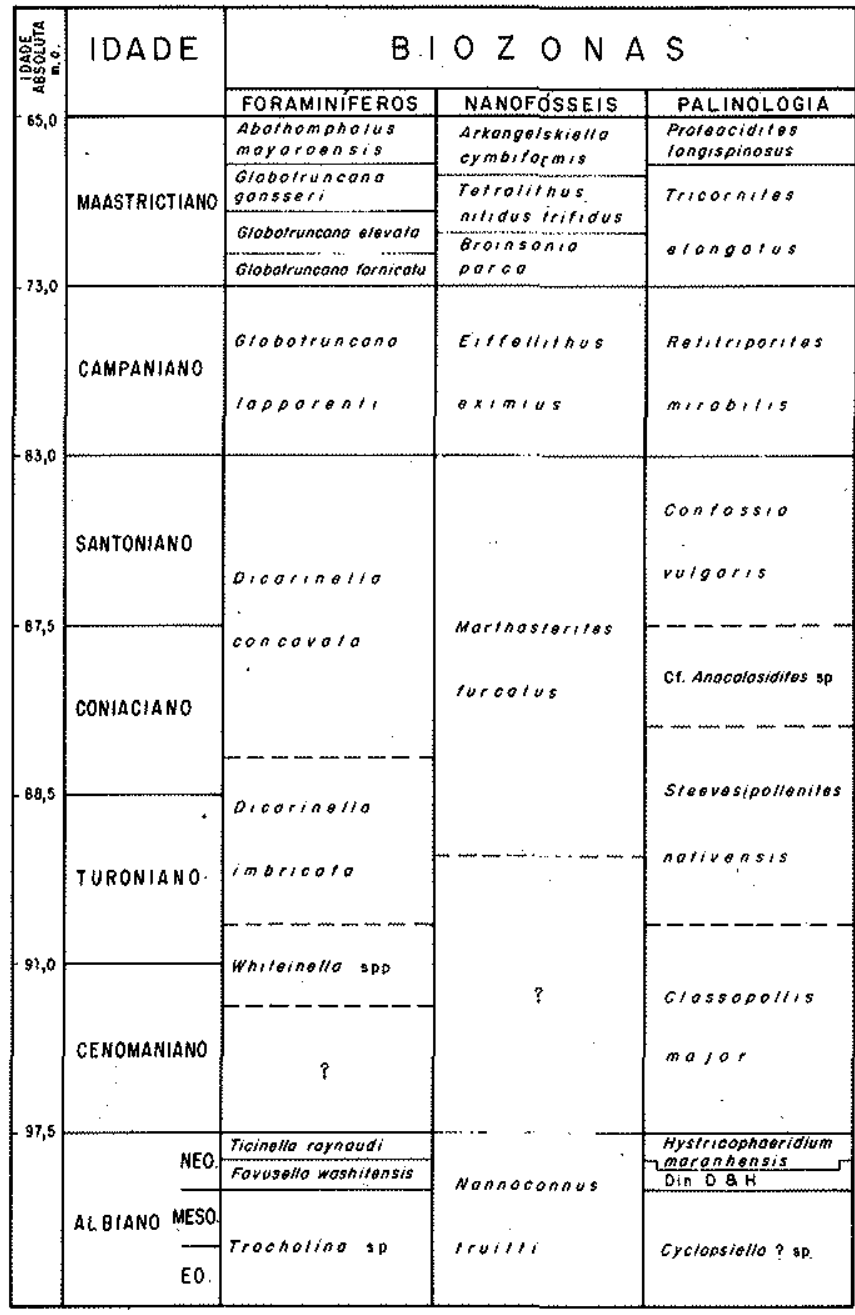

Figura 3 - Posicionamento das biozonas na coluna cronogeológica

guti \& Santos (1972), cujo nome foi alterado, mas esta biozona não atinge os estratos mais superiores do Neoalbiano na Bacia de Campos, com a extinção da forma-guia ocorrendo num nível estratigráfico inferior ao do gênero Ticinella.

$\mathrm{O}$ poço $7 \cdot \mathrm{CH}-2-\mathrm{RJS}$ detém a seção mais representativa desta biozona.

ZONA Ticinella raynaudi Esta biozona representa o pacote de rocha depositado entre o instante de desaparecimento da espécie Favusella washitensis e do gênero Ticinella, mais destacadamente das espécies $T$. raynaudi e T. primula.

A extinção do gênero Ticinella no final do Albiano é reconhecida por alguns autores (Ascoli 1976, Van Hinte 1976). A biozona representa, portanto, o desfecho da sedimentação albiana na bacia e seu topo corresponde aos das zonas Nannoconus truitti, de nanofósseis, Hystricosphaeridium maranhensis, de palinomorfos, e a extinção dos calcisferulídeos na bacia.

Também no poço 7-CH-2-RJS é registrada a melhor seção desta biozona.
ZONA Whiteinella ssp. (INFORMAL) Trata-se de uma zona-intervalo cujo topo corresponde ao nível de extinção local do gênero Whiteinella. Neste horizonte estratigráfico desaparecem também os bentônicos Globorotalites michelinianus e Dorothia oxycona, e uma espécie de radiolário bem distinta: Amphibrachium leptum. As espécies Praeglobotruncana stephani e $P$. gibba, nos poucos poços em que foram reconhecidas, não ultrapassam igualmente este bio-horizonte. Raras são também as hedbergelas em estratos sotopostos a este nível.

Vários pesquisadores descrevem, na região-tipo do Turoniano e vizinhanças, um intervalo rico em planctônicos globosos, informalmente designado por zone à grandes globigerines, que se estende da parte mais superior do Cenomaniano à parte inferior do Turoniano (Lehmann 1962, Robaszynski 1979, Robaszynski et al. 1980, Robaszynski et al. 1982, entre outros). Trata-se de uma camada onde as whiteinelas são bastante freqüentes, embora não corresponda a seu horizonte estratigráfico final de ocorrência. A biozona reconhecida na Bacia de Campos exibe uma boa correspondência com este estrato, contendo razoável número de formas arredondadas (Whiteinella e Hedbergella) e uma associação específica indicativa do Neocenoma-

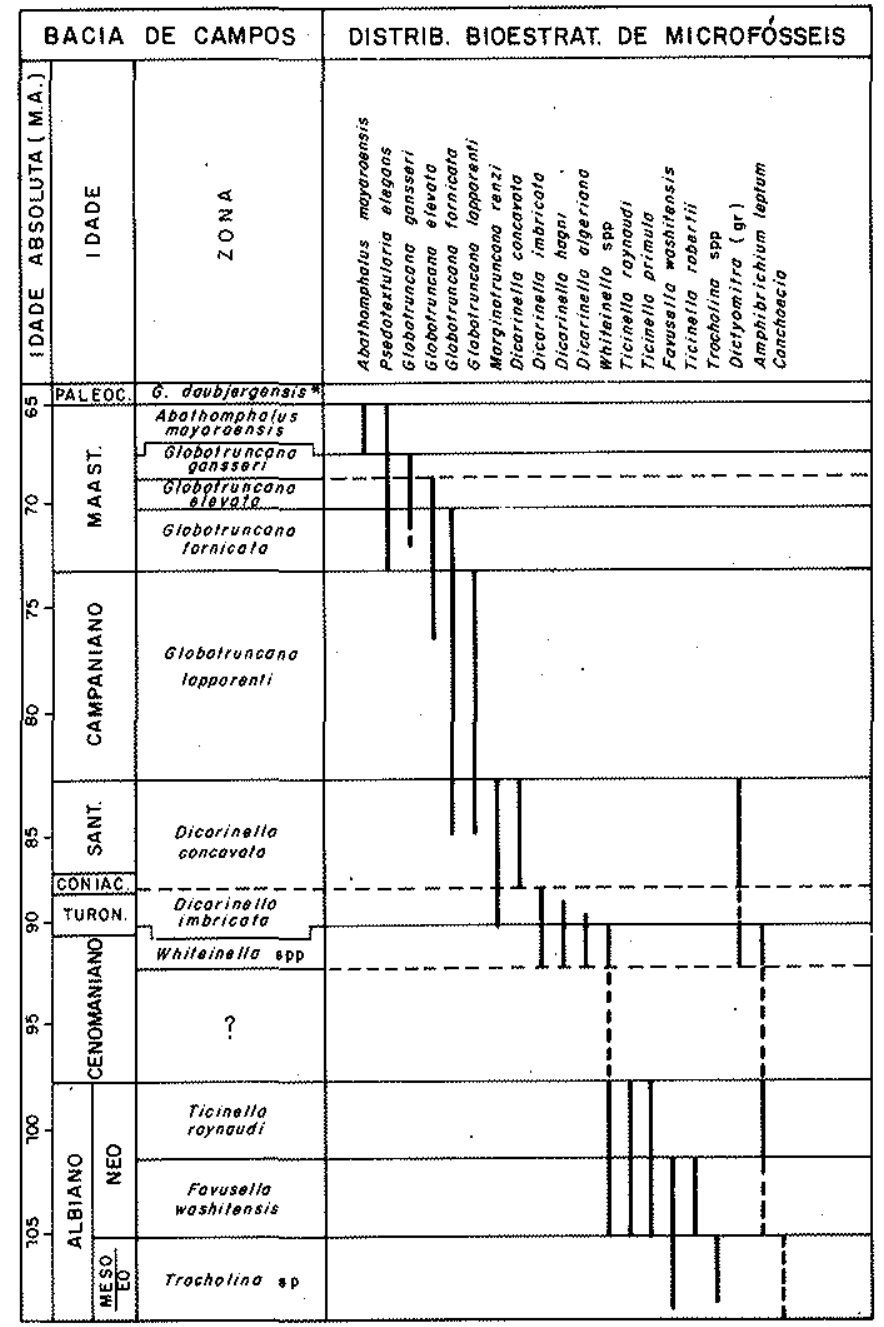

* Zono nõo objeto deste estudo

Figura 4 - Distribuição bioestratigráfica de microfósseis 
niano-Eoturoniano.

Os poços 1-RJS-90 e 1-RJS-27 são os que melhor ilustram esta unidade bioestratigráfica na Bacia de Campos.

ZONA Dicarinella imbricata (INFORMAL) Trata-se de uma biozona pouco freqüente em Campos e, por isso, classificada informalmente. Seu limite superior é definido ao nível de extinção da espécie $D$. imbricata e sua base coincide com o topo da zona anterior.

Cronoestratigraficamente associada ao Turoniano-Conia* ciano (parte inferior) a partir da amplitude de ocorrência da forma diagnóstica (Rodríguez 1977, Robaszynski \& Caron 1979), esta biozona inclui ainda dois bons fósseis-guia - os taxa $D$. algeriana e $D$. hagni, que foram considerados como formas associadas.

A região central da bacia concentra os registros desta biozona, ocorrendo no poço 1-RJS-184 sua melhor seção.

ZONA Dicarinella concavata Esta unidade bioestratigráfica é definida pelos níveis de extinção dos planctônicos $D$. imbricata e $D$. concavata ou Marginotruncana renzi.

Existem pesquisadores que posicionam o horizonte de desaparecimento da $D$. concavata mais tardiamente que o da espécie $M$. renzi (Robaszynski \& Caron 1979, Ascoli 1976, Postuma 1971). Os resultados obtidos, no entanto, não dão suporte para tal distinção. Assim, uma única biozona, amplamente distribuída na bacia, representa o intervalo Coniaciano (parte superior) -Santoniano.

Os poços 1-RJS-1.38 e 1-RJS-30 representam relativamente bem esta biozona.

ZONA Globotruncana lapparenti (INFORMAL) Esta biozona tem seu limite de ocorrência definido pela extinção da espécie $G$. lapparenti. e estende-se até o topo da zona $D$. concavata.

Embora exista uma grande controvérsia quanto ao nível de desaparecimento da espécie diagnóstica e não ocorra na seção a Zona-amplitude G. calcarata, excelente horizonte referência mundial para a porção superior do Campaniano (Longoría 1984), a zona $G$. lapparenti foi relacionada com este andar face à correspondência demonstrada com a Zona Eiffellithus eximius, de nanofósseis. Optou-se, no entanto, por uma designação informal devido à escassez de registro desta unidade na bacia. Sua melhor seção foi recuperada no poço 1-RJS-66.

ZONA Globotruncana fornicata Esta unidade estratigráfica foi incluída no zoneamento de Noguti \& Santos (1972). Propõe-se apenas a redefinição de seu limite inferior, posicionando-o no nível de extinção da espécie $G$. lapparenti.

Cronoestratigraficamente, esta biozona limita-se ao Maastrichtiano Inferior, com a a extinção da $G$. fornicata. Sua melhor seção foi registrada no poço 1-RJS-52.

ZONA Globotruncana elevata (INFORMAL) Com os limites demarcados nos níveis de extinção das espécies $G$. fornicata e $G$. elevata, esta biozona foi registrada com mais frequência na área centro-oeste da bacia destacadamente no poço 1-RJS-205.

Embora alguns autores estendam a amplitude de ocorrência da forma-guia ao final do Maastrichtiano, observa. ções feitas na área de Campos indicam que esta espécie não alcança o nivel de extinção do nanofóssil Tetralithus nitidus trifidus, verificado no Neomaastrichtiano. Resultado similar foi alcançado por Rodríguez (1977).

ZONA Globotruncana gansseri Esta bio-unidade foi identificada na Bacia do Ceará por Viviers (1982). Em Campos, compreende o pacote sedimentar depositado entre os níveis de desaparecimento das espécies $G$. elevata e $G$. gansseri.

Reconhecida mundialmente, essa zona é associada ao Neomaastrichtiano. $O$ melhor poço de referência na bacia é o 1-RJS-3B.

ZONA Abathomphalus mayaroensis (INFOR-

$M A L)$ Com uma distribuição bem deficiente, esta unidade bioestratigráfica foi sugerida em razão da diminuta amplitude temporal da espécie-guia, relacionada globalmente com o final do Maastrichtiano (Pessagno 1967).

Espécimes de $A$. mayaroensis foram encontrados em poucos poços, deslocados de seu real horizonte estratigráfico (exemplo 1-RJS-3B). Por isso optou-se por incluir esta bio-unidade de maneira informal.

Nanofósseis A figura 5 apresenta a distribuição das ZONA Nannoconus truitti Definida por Troelsen \& Quadros (1971), esta biozona tem sua distribuição

ZONA Nannoconus truitti Definida por Troelsen \& Quadros (1971), esta biozona tem sua distribuição temporal limitada ao Albiano na área de Campos, já que o horizonte de extinção de sua forma-guia coincide com o da Ticinella raynaudi.

Essa unidade bioestratigráfica distribui-se por toda a bacia e é bem representada no poço 7-CH-2,RJS.

INTER VALO INDETERMINADO Na seção correspondente às zonas Classopolis major e Whiteinella spp., constata-se uma associação de nanofósseis pouco diversificada, constituída, basicamente, pelas espécies Watznaueria barnesare e Eiffellithus turriseiffeli. Esta zona-intervalo é de difícil reconhecimento em amostras de calha, motivo pelo qual não foi proposta neste trabalho.

ZONA Marthasterites furcatus Trata-se de uma unidade bioestratigráfica que substitui a da zona Lithastrinus grillii de Troelsen \&Quadros, (1971), procurando ressaltar a ocorrência da espécie $M$. furcatus, fóssil-guia do Coniaciano-Santoniano (Thierstein 1976, Proto-Decima et al. 1978).

Seus limites são definidos superiormente no horizonte de desaparecimento das espécies Lithastinus grillii, L. floralis e Masthasterites furcatus. Sua base é relacionada com a primeira aparição do $L$. grillii. $\overrightarrow{\mathrm{E}}$ uma zona bem comum na bacia e encontra-se bem representada no poço 1-RJS-193.

ZONA Eiffellithus eimius Esta unidade bioes. tratigráfica foi reconhecida, pela primeira vez no Brasil, por Freitas (1984) na Bacia Sergipe-Alagoas. Corresponde à Zona Eiffellithus augustus, definida por Antunes (1984), cujo nome foi considerado impróprio.

Reconhecida em vários outros pontos do globo (Thierstein 1976), a espécie-guia tem sua extinção relacionada com o topo do Campaniano. Em Canpos, sua distribuição é bastante limitada, concentrando-se na área central da bacia. $O$ 


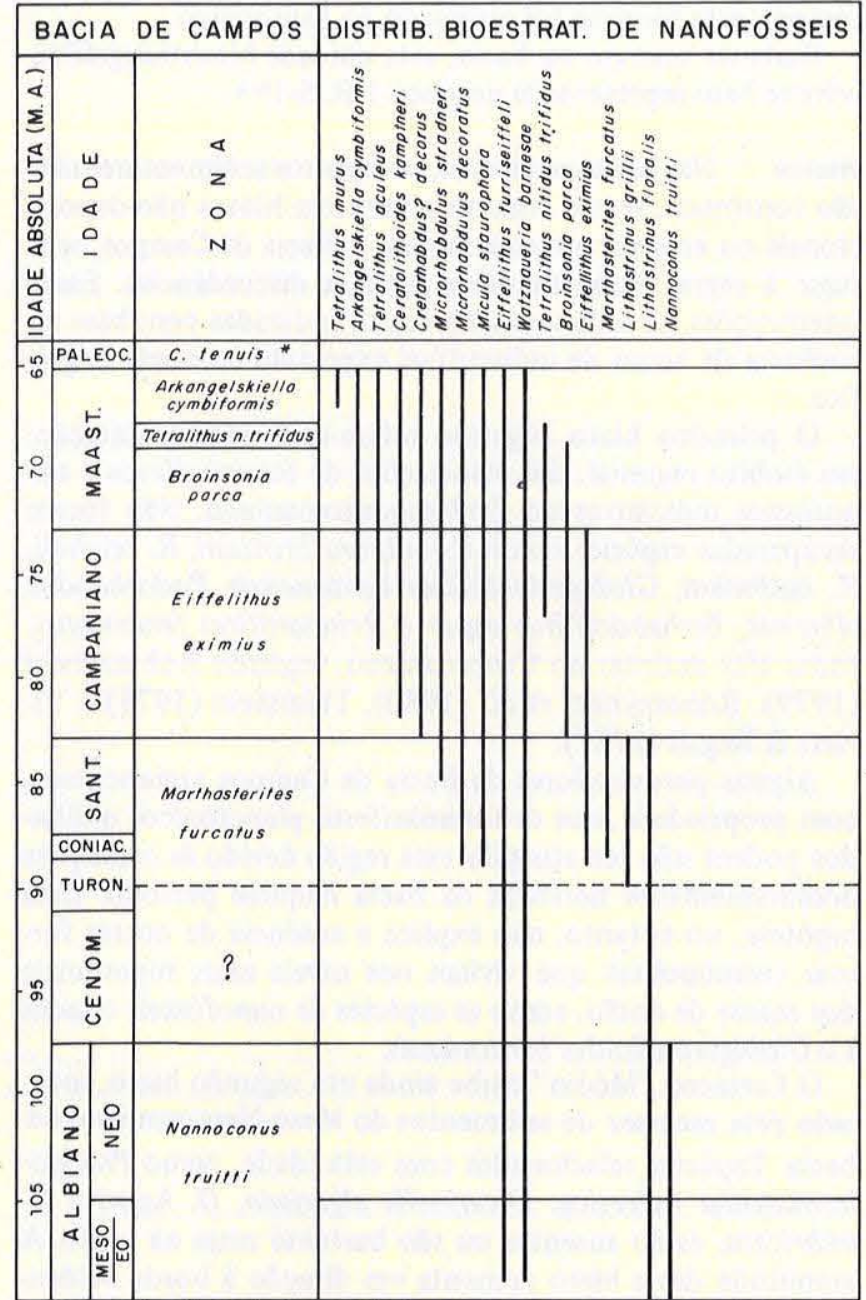

* Zona năo objeto deste estudo

Figura 5 - Distribuição bioestratigráfica de nanofósseis

poço 1-RJS-184 ilustra com propriedade esta biozona.

ZONA Broinsoni parca Definida por Troelsen \& Quadros (1971), esta biozona foi, durante muito tempo, tida como de idade campaniana. Estudos mais recentes, no entanto, têm demonstrado a presença de Broinsonia parca em estratos maastrichtianos. Na área em questão, seu desaparecimento ocorre após a extinção do planctônico Globotruncana fornicata, datada como Eomaastrichtiano.

Embora não muito freqüente, esta biozona tem sido encontrada na bacia e está bem representada no poço 1-RJS-205.

ZONA Tetralithus nitidus trifidus Trata-se de mais uma zona emendada por Troelsen \& Quadros, em 1971. Cronoestratigraficamente, teve seu topo posicionado no Neomaastrichtiano.

Pouco freqüente na bacia, esta biozona acha-se bem representada no poço 1-RJS-3B.

ZONA Arkangelskiella cymbiformis Proposta por Troelsen \& Quadros (1971), esta unidade bioestratigráfica estabelece o topo do Maastrichtiano e distribuiu-se amplamente na região.

Palinologia A figura 6 resume a distribuição bioestratigráfica de palinomorfos na Bacia de Campos.

ZONA Cyclopsiella sp. (INFORMAL) Esta biozona contém os mais antigos fósseis marinhos da bacia. Seus limites são definidos pelos níveis de surgimento e extinção da forma Cyclopsiella sp., um incertas sedis anteriormente designado por Uesugui (1976) como Acritarcha (?) correspondendo ao "intervalo $\alpha$ " deste autor.

Originalmente relacionada com o Eoalbiano, esta zona foi estendida ao Mesoalbiano após o estudo de calcisferulídeos feito por Dias-Brito em 1985.

ZONA DIN. D/DIN. H (INFORMAL) Trata-se de uma zona diferencial superior com limites estabelecidos nos níveis de desaparecimento das formas Cyclopsiella sp. e Din $\mathrm{D}$ e/ou Din $\mathrm{H}$, que são tipos de dinoflagelados ainda não formalmente descritos.

Esse pacote rochoso foi posicionado cronoestratigraficamente no Neoalbiano, estando correlacionado à Zona $\mathrm{Fa}$ vusella washitensis de foraminíferos.

O poço 1-RJS-19 é o que melhor registra esta biozona na bacia.

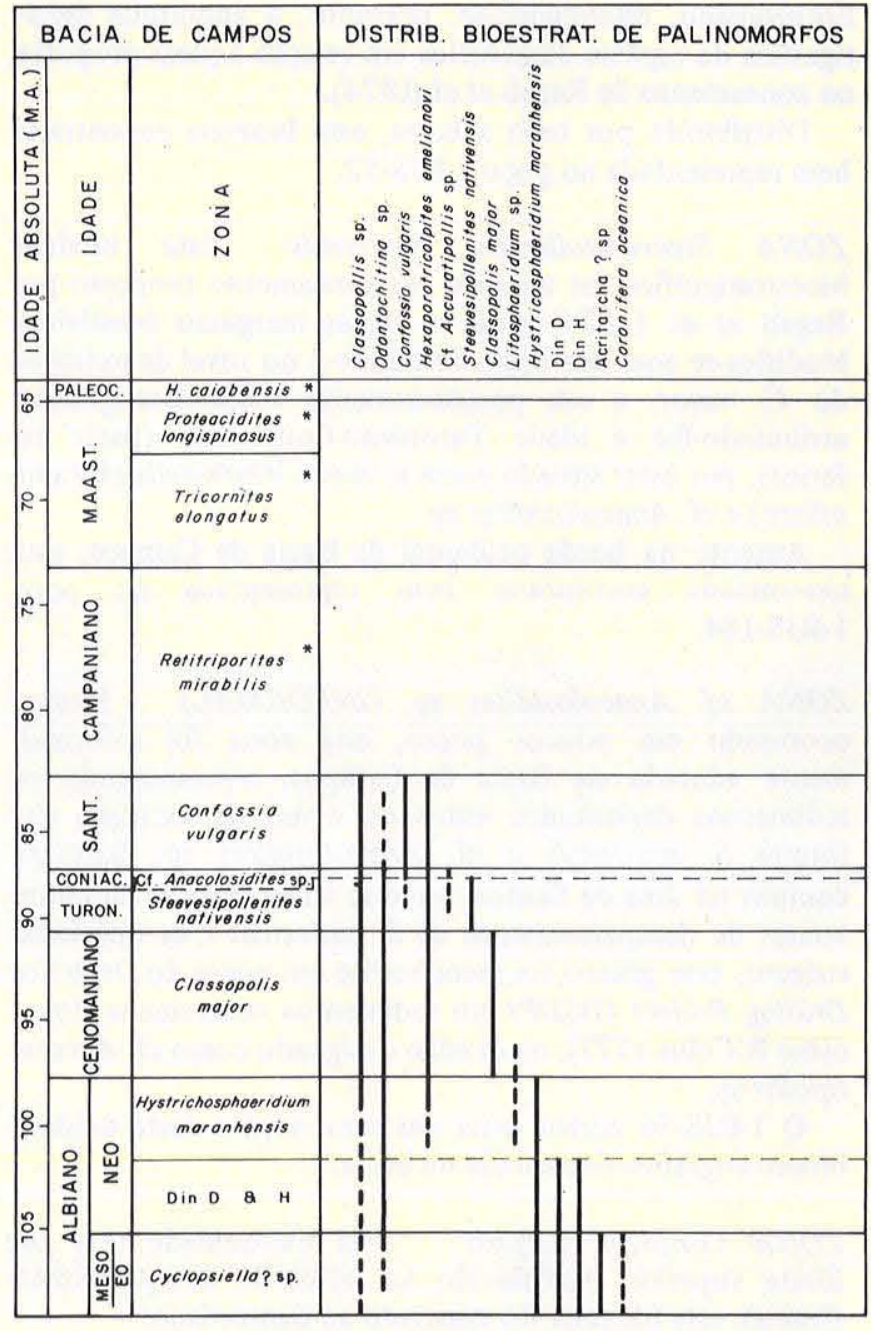

* Zona nāo objeto de esfudo

Figura 6 - Distribuição bioestratigráfica de palinomorfos 
ZONA Hystrichosphaeridium maranhensis Esta unidade bioestratigráfica tem seu limite superior definido pela extinção de $H$. maranhensis e o inferior marcado no nível de desaparecimento dos dinoflagelados D e H. Corresponde ao "intervalo $\beta$ " de Uesugui (1976).

Com seu topo estratigráfico coincidente com as zonas Ticinella raynaudi, de foraminíferos, Nannoconus truitti, de nanofósseis, e Pithonella sphaerica-Pithonella ovalis-Bonetocardiella conoidea, de calcisferulídeos, esta biounidade foi associada ao final do Albiano.

Sua distribuição na bacia é bastante ampla, sendo reconhecida uma boa seção no poço 7-CH-2-RJS.

ZONA Classopolis major Esta biozona tem seu limite associado à extinção de $C$. major e estende-se até o topo da zona $H$. maranhensis.

Correspondendo ao "intervalo $\gamma$ " de Uesugui (1976), esta unidade bioestratigráfica foi, por este autor, relacionada com o Neoalbiano-Cenomaniano. Com o exame dos calcisferulídeos da Bacia de Campos, a biozona $C$. major foi definitivamente desvinculada do Albiano e associada duvidosamente ao Cenomaniano.

Com o estudo dos foraminíferos planctônicos presentes neste intervalo, constatou-se certa correspondência entre o topo dessa unidade e o da Zona Whiteinella spp. Assim, a Zona Classopolis major foi relacionada ao NeocenomanianoEoturoniano, estendendo-se, portanto, a amplitude estratigráfica da espécie diagnóstica em relação àquela proposta no zoneamento de Regali et al. (1974).

Distribuída por toda a bacia, esta biozona encontra-se bem representada no poço 1-RJS-52.

ZONA Steevesipollenites nativensis Esta unidade bioestratigráfica foi incluída no zoneamento proposto por Regali et al. (1974) para as bacias marginais brasileiras. Modifica-se aqui sua base, definindo-a no nível de extinção do $C$. major, e seu posicionamento cronoestratigráfico, atribuindo-lhe a idade Turoniano-Coniaciano (parte inferior), por estar situado entre as zonas Whiteinella (foraminífero) e cf. Anacolosidites sp.

Ausente na borda ocidental da Bacia de Campos, esta bio-unidade encontra-se bem representada no poço 1-RJS-184.

ZONA cf. Anacolosidites sp. (INFORMAL) Mesmo ocorrendo em poucos poços, esta zona foi informalmente adotada na Bacia de Campos, representando os sedimentos depositados entre os níveis de extinção das formas $S$. nativensis e cf. Anacolosidites sp. Bastante comum na área de Santos, onde se situa entre os bio-horizontes de desaparecimento de $S$. nativensis e de Confossia vulgaris, este gênero foi reconhecido em poços do Deep Sea Drilling Project (DSDP) em sedimentos coniacianos (Ioannides \& Colin 1977), na ocasião designado como cf. Accuratipollis sp.

O 1-RJS-56 cortou uma das raras seções desta unidade bioestratigráfica depositada na bacia.

ZONA Confossia vulgaris Esta bio-unidade teve seu limite superior estabelecido ao nível de desaparecimenfósseis), esta biozona foi associado ao Santoniano. zona cf. Anacolosidites sp.

Tendo seu topo relacionado com as zonas Dicarinella concavata (foraminíferos) e Marthasterites furcatus (nano- fósseis), esta biozona foi associado ao Santoniano.

Bastante comum na bacia, esta unidade bioestratigráfica acha-se bem representada no poço 1-RJS-193.

Hiatos Nas bacias oceânicas, os registros sedimentares não são contínuos, sendo comum ocorrerem hiatos não-deposicionais ou erosivos em suas seções. A Bacia de Campos, sem fugir à regra, exibe também algumas discordâncias. Essas interrupções na sedimentação foram indicadas com base na ausência de zonas de indiscutível expressão cronoestratigráfica.

$\mathrm{O}$ primeiro hiato sugerido refere-se à não-constatação, em âmbito regional, das associações de foraminíferos e nanofósseis indicativas do Eo-Mesocenomaniano. Não foram recuperadas espécies como Rotalipora brotzeni, $R$. reicheli, R. cushmani, Globigerinelloides bentonensis, Podorhabdus albianus, Parhabdolithus asper e Reinhardtites fenestratus, todas elas extintas no Cenomaniano, segundo Robaszynski (1979), Robaszynski et al. (1980), Thierstein (1976) e Viviers \& Regali (1987).

Alguns pesquisadores da Bacia de Campos argumentam, com propriedade, que os foraminíferos planctônicos quilhados podem não ter atingido esta região devido às condições dominantemente neríticas da bacia naquele período. Esta hipótese, no entanto, não explica a ausência de outras formas cosmopolitas que viviam nos níveis mais superficiais dos mares de então, como as espécies de nanofósseis citadas e o Globigerinelloides bentonensis.

O Cretáceo "Médio" exibe ainda um segundo hiato, indicado pela escassez de sedimentos do Meso-Neoturoniano na bacia. Espécies relacionadas com esta idade, como Praeglobotruncana helvetica, Dicarinella algeriana, $D$. hagni e $D$. imbricata, estão ausentes ou são bastante raras na seção. A amplitude deste hiato aumenta em direção à borda ocidental da bacia, onde não ocorre a Zona Steevesipollenites nativensis correspondente ao Neoturoniano-Coniaciano (parte inferior).

A última das megadescontinuidades deposicionais é indicada pela restrita distribuição em área das bio-unidades relacionadas com o Campaniano. Os núcleos representativos deste andar concentram-se na região central da bacia e em pontos isolados na porção norte. Já o Andar Maastrichtiano distribuiu-se de modo mais contínuo em Campos, mas também exibe discordâncias menos expressivas em seu interior.

CONCLUSÕES Um arcabouço biocronoestratigráfico para a Bacia de Campos é apresentado pela primeira vez, incluindo informações quanto à sucessão dos foraminíferos, nanofósseis e palinomorfos em sua seção marinha cretácea.

Ao todo, foram reconhecidas 27 biozonas, sendo 11 de foraminíferos, 10 de palinomorfos e seis de nanofósseis. Algumas dessas unidades têm caráter informal, mas contribuem para dividir a seção Albiano-Maastrichtiano em 16 bio-horizontes, que foram tentativamente relacionados com a escala de tempo absoluta.

Cronoestratigraficamente, a seção apresenta algumas descontinuidades, sendo raros os registros de sedimentos do Turoniano Médio-Superior e Campaniano. Foi também sugerida a ausência do Cenomaniano Inferior/Médio na bacia, posto que não foram reconhecidas as zonas de foraminíferos internacionalmente indicativas deste intervalo. Hiatos menores são identificados no Coniaciano e no Maastrichtiano.

Não foi possível atingir um grau de detalhamento unifor- 
me para todos os andares. Se o Albiano, o Coniaciano e o Maastrichtiano foram suficientemente fatiados, o mesmo não se pode dizer em relação ao Santoniano e aos isolados núcleos do Campaniano.

Agradecimentos Os autores expressam seus agradeci- mentos a todos os geólogos do Setor de Bioestratigrafia e Paleoecologia do Cenpes, que prestaram seu apoio e contribuição a este trabalho; e à diretoria da Petrobrás, pela liberação do trabalho para publicação.

\section{REFERENCIAS BIBLIOGRAFICAS}

ANTUNES, R.L. - 1984 - Geohistória do Paleocanyon de Fazenda Cedro, Bacia do Espírito Santo, Brasil, segundo dados bioestratigráficos. In: CONGR. BRAS. GEOL, 33, Rio de Janeiro, 1984. Anais... Rio de Janeiro, SBG. v.2, p. 670-684.

ASCOLI, P. - 1976 - Foraminiferal and ostracod biostratigraphy of the Mesozoic-Cenozoic, Scotian Shelf, Atlantic Canada. In: SYMP. BENTHONIC FORAMINIFERAL OF CONTINENTAL MARGINS, 1, S.L., 1976. Proceedings... s.l. p. 653-771 (Part B, Paleocology and Bioestratigraphy, Maritime Sediments Spec. Publ. 1).

AZEVEDO, R.L.M. de; GOMIDE, J.; VIVIERS, M.C. - 1987 Geohistótia da Bacia de Campos, Brasil: do Albiano ao Maastri* chtiano. Rev. Bras. Geoc., 17(2): 139-146.

BEURLEN, G. - 1982 -- Bioestratigrafia e geohistória da seção marinha da Margem Continental Brasileira. Bol. Tec. Petrobrás, 25(2):77-83.

DIAS-BRITO, D. - 1985 - Calcisphaerulidae do Albiano da Bacia de Campos - Rio de Janeiro - Brasil: investigações taxonômicas, biocronoestratigráficas e paleoambientais. In: Brasil, MMEDNPM: Col. Trab. Paleont., Brasília, DNPM. p. 295-305 (Geologia 27, Paleontologia e Estratigrafia 2).

FIGUEIREDO, A.M.F. de \& MOHRIAK, W.U. - 1984 - A tectônica salífera e as acumulações de petróleo da Bacia de Campos. In: CONGR. BRAS. GEOL., 33, Rio de Janeiro, 1984. Anais.. Rio de Janeiro, SBG. v. 3, p. 1380-1394.

FREITAS, L.C.S. - 1984 -Nanofósseis calcários e sua distribuição (Aptiano-Mioceno) na Bacia de Sergipe-Alagoas. Rio de Janeito, 256 p. (Dissertação de Mestrado, UFRJ).

HARLAND, W.B.; COX, A.V.; LLEWELLYN, P.G.; PICKTON, C.A.G.; SMITH, A.G.; WALTERS, R - 1982 - A Geologic time scale. 1st. ed., Cambridge, Cambridge University Press. $131 \mathrm{p}$.

IOANNIDES, N.S. \& COLIN, J.P. - 1977 - Palynology of sites 358 356, 355 DSDP, Leg 39, Southwestern Atlantic Ocean. In: SUPKO, P.R. \& PERCH-NIELSEN, K. Inst. Repts. DSDP, 39, Washington (U.S. Govt. Printing Office), p. 285-297.

LEHMANN, R. - 1962 - Étude des Globotruncanides du Crétacé Supérieur de la Province de Tarfaya (Maroc. Occidental). Notes Serv. Geol. Maroc., 21(1.56):133-159.

LONGORIA, J.F. - 1984 - Cretaceous biochronology from the Gulf of Mexico region based on planktonic microfossils. Micropaleontology, 30(3):225-245.

NOGUTI, I. \& SANTOS, J.F. - 1972 - Zoneamento preliminar por foraminíferos planctônicos do Aptiano ao Mioceno na Plataforma Continental do Brasil. Bol. Téc. Petrobrás, 15(3): 265-283.

PESSAGNO, Jr., E.A. - 1967 - Upper Cretaceous planktonic foraminifera from the Western Gulf Coastal Plain. Paleontographica Americana, 5(37):243-445.

POSTUMA, J.A. - 1971 - Manual of planktonic foraminifera. Amsterdam, Elsevier. $420 \mathrm{p}$.

PROTO-DECIMA, F; MEDIZZA; F; TODESCO, F. - 1978 Southeastern Atlantic Leg 40, calcareous nannofossils. In: BOLLI, H.M. \& RYAN, W.B.F., Init. Repts. DSDP, 40, Washington (U.S. Govt. Printing Office), p. 571-635.

QUADROS, L.P. \& GOMIDE, J. - 1972 - Nanofósseis calcários na Plataforma Continental Brasileira. Bol. Téc. Petrobrás, 15(4):339-354.
REGALI, M.S.P.; UESUGUI, N.; SANTOS, A.S. - 1974 - Palinologia dos sedimentos Meso-Cenozóicos đo Brasil (I). Bol. Téc. Petrobrás, 17(3):177-191 e 17(4):236-301.

ROBASZYNSKI, F. - 1979 - Comparison between the Middle Cretaceous of Belgium and some French Regions. In: Aspekte der Kreide Europas, I.U.G.S. p. 543-561 (Series A, 6).

ROBASZYNSKI, F, \& CARON, M. (coord.) - 1979 - Atlas de foraminiferes planctoniques du Crétacé Moyen (Mer Boréale et Téthys). Paris, CNRS. v. 1, p. 1-185; v. 2, p.1.181 (Parts 1-2, Cah. micropaceont.)

ROBASZYNSKI, F. \& AMEDRO, F. (coord.) - 1980 - Synthèse biostratigraphique de l'Aptian au Santonian du Boulonnais à partir de sept groupes paléontologiques: foraminifères, nannoplancton, dinoflagellés et macrofau nes. Rev. Micropaleont., 22(4):195-321.

ROBASZYNSKI, F.; AMEDDRO, F.; FOUCHER, J.C.; GASPARD, D.; MAGNIEZ-JANNIN, F.; MANIVIT, H.; SORNAY, J. 1980 - Synthèse bioestatigraphique de l'Aptian au Santonian du Boulonnais à partir de sept groupes paléontologiques: foraminifères, nannoplancton, dinoflagellés et macrofaunes. Rev. Micropaléont., 22(4):195-321.

RODRIGUEZ, D.L. - 1977 - Estudio de los foramintferos planctónicos del Cretácico Superior de las Cordillezas Béticas (Sector Central). Málaga (Tese de Doutorado) Univ. de Málaga, Espanha, 410 p., $148 \mathrm{pl}$.

SCHALLER, H. - 1973 - Estratigrafia da Bacia de Campos. In CONGR. BRAS. GEOL., 27, Aracajú, 1972. Anais... Aracajú, SBG. v. 3, p. 247-258.

THIERSTEIN, H.R. - 1976 - Mesozoic calcareous nannoplankton biostratigraphy of marine sediments. Mar. Micropaleont. 1 (1986) p. 325-362.

TROELSEN, J.C. \& QUADROS, L.P. - 1971 - Distribuição bioestratigráfica dos nanofósseis em sedimentos marinhos (Aptiano-Mioceno) do Brasil. An. Acad. bras. Ciênc., 43:577-609.

UESUGU1, N. - 1976 - Intervalos bioestratigráficos da Bacia de Campos do Albiano ao Santoniano. Rio de Janeiro, Petrobrás (rel. int.).

VAN HINTE, J.E. - 1976 - A cretaceous time scale. AAPG Bull., 60(4):498-516

VIVIERS, M.C. - 1982 - Biocronoestratigrafia da Bacia do Ceará. In: CONGR. BRAS. GEOL., 32, Salvador, 1982, Anais... Salvador, SBG. v. 5 ; p. 2433-2449.

VIVIERS, M.C. \& REGALI, M.S.P. - 1987 - Estudo paleoambiental preliminar no Cretáceo da Bacia Potiguar. Rev. Bras. Geoc., 17(2): $123-130$.
MANUSCRTTO 401

Recebido em 31 de Janeiro de 1985 Revisão aceita em 01 de Abril de 1987 